\title{
On the Volume Formula for Hyperbolic Tetrahedra*
}

\author{
Yunhi Cho and Hyuk Kim \\ Department of Mathematics, Seoul National University, \\ Seoul 151-742, Korea \\ \{yhcho, hyukkim\}@math.snu.ac.kr
}

\begin{abstract}
We present a volume formula for arbitrary hyperbolic tetrahedra and give applications for Coxeter tetrahedra.
\end{abstract}

\section{Introduction}

In Euclidean, hyperbolic, or spherical space, polyhedra are fundamental geometric objects, and calculating the volume of a convex polyhedron is a very old but difficult geometric problem in general. Due to the recent progress in hyperbolic manifold theory, this problem has become more interesting to the wider mathematical community. As is well known, every hyperbolic manifold can be obtained by identifying the faces of a convex polyhedron in the hyperbolic space. Its volume is a topological invariant by Mostow rigidity when the dimension is greater than or equal to three and hence is an important invariant in the classification of hyperbolic manifolds. Volume computation based on the dihedral angles is especially more useful in this and the theory of discrete subgroups of hyperbolic isometry groups.

Since a polyhedron can be divided into simplices by simplicial subdividion, the first step is to consider the volume of a simplex and it is our intention in this paper to investigate the problem of finding a volume formula for a three-dimensional hyperbolic simplex. Note that the volume formula of a spherical simplex only differs from that of a hyperbolic one by a universal constant (see [2] and [11]), and we are concerned only with hyperbolic volumes in this paper.

In Euclidean space, the volume of an $n$-simplex is obtained from the determinant of the vectors determined by its vertices.

\footnotetext{
* This work was supported (in part) by the Korean Science and Engineering Foundation (KOSEF) through the GARC at Seoul National University.
} 
In non-Euclidean space, the volume of tetrahedra cannot be expressed so easily in terms of its dihedral angles. In the first half of the nineteenth century Lobachevsky obtained a formula that expressed the volume of a hyperbolic 3-orthoscheme (see Section 3.1) as a function of its dihedral angles using a special function known (essentially) as the Lobachevsky function. Since any tetrahedron can be represented as an algebraic sum of orthoschemes, this formula in principle is sufficient to determine the volume of any tetrahedron. However, we have to determine new dihedral angles which occur in the dissection process of the tetrahedron into the orthoschemes, and the problem of finding a volume formula gets much more complicated and is difficult to write down in a closed form.

For an ideal tetrahedron, Milnor observed [7] that the volume formula can be expressed in a very simple way as the sum of the values of the Lobachevsky function of the dihedral angles at any vertex. In general there is a simple closed volume formula for a tetrahedron with at least one vertex at infinity, due to Vinberg. In fact, he obtained a volume formula of $n$-pyramid with apex at infinity [10]. However, for compact polyhedra, there are not many examples whose closed volume formulas are known (see [4] and [5]). Particularly, in [4] Kellerhals showed that the volume formulas of 1-truncated or 2-truncated orthoschemes are the same as the volume formulas of the orthoschemes. She also found the volume formula of Lambert cubes.

The purpose of this paper is to give another and special method to derive a volume formula for arbitrary simplices. The method is combinatorial in nature and does not involve dissections of the simplex or Schläfli volume differential. We examine the configuration of the ideal polyhedra determined by the faces of the given simplex and observe that a suitable combination of these polyhedra gives the volume of our simplex. Of course, the volume of these ideal ployhedron can then be computed using Milnor's formula. Then we deduce the Lobachevsky formula for 3-orthoschemes and two unknown simple formulas for special types of tetrahedra from the general formula. Meyerhoff [6] used orthoscheme decomposition to determine the volumes of all Coxeter tetrahedra in numerical values only. In the Appendix we give simple exact representations of volumes of all Coxeter tetrahedra in terms of the Lobachevsky function, using our formula.

\section{Lobachevsky Function}

\subsection{Lobachevsky Function}

The volumes of the polyhedra in three-dimensional hyperbolic space $\mathbb{H}^{3}$ are expressed by Lobachevsky functions of dihedral angles. We define the Lobachevsky function denoted by $\wedge$ by the formula

$$
\wedge(x):=-\int_{0}^{x} \log |2 \sin t| d t, \quad x \in \mathbb{R} .
$$

The function $\wedge$ is odd, periodic with period $\pi$, vanishes at points $n \pi / 2$, and it takes its maximum value at $n \pi+\pi / 6$, and its minimum value at $n \pi-\pi / 6$. 
For each natural number $n, \wedge(x)$ satisfies the following functional equation:

$$
\wedge(n x)=n \sum_{k=0}^{n-1} \wedge\left(x+\frac{k \pi}{n}\right) .
$$

In particular, for $n=2$, this relation yields

$$
\wedge(2 x)=2 \wedge(x)+2 \wedge\left(x+\frac{\pi}{2}\right) .
$$

One can see more properties of the function $\wedge(x)$ in [7] and [4].

\subsection{Gram Matrix}

We use the hyperboloid model for $\mathbb{H}^{3}$ in what follows, that is, $\mathbb{H}^{3}$ is embedded in the Lorentz space $\mathbb{R}^{1,3}$ of signature $(1,3)$. Here $\mathbb{R}^{1,3}$ denotes the real vector space $\mathbb{R}^{4}$ equipped with the bilinear form of signature $(1,3)$ :

$$
\langle x, y\rangle=-x_{0} y_{0}+x_{1} y_{1}+x_{2} y_{2}+x_{3} y_{3}
$$

for all $x=\left(x_{0}, x_{1}, x_{2}, x_{3}\right)$ and $y=\left(y_{0}, y_{1}, y_{2}, y_{3}\right) \in \mathbb{R}^{4}$. Hence $\mathbb{H}^{3}$ can be identified with

$$
\left\{x \in \mathbb{R}^{1,3} \mid\langle x, x\rangle=-1, x_{0}>0\right\} .
$$

An extensive discussion of the properties of this model can be found in [1] and [9].

Let $P \subset \mathbb{H}^{3}$ denote a convex polyhedron with finitely many faces $F_{i}, i \in I$, which are characterized by unit normal vectors $e_{i} \in \mathbb{R}^{1,3}$ directed outward with respect to $P$. Then we have

$$
F_{i} \subset e_{i}^{\perp}:=\left\{x \in \mathbb{R}^{1,3} \mid\left\langle x, e_{i}\right\rangle=0\right\} .
$$

The Gram matrix $G(P):=\left(\left\langle e_{i}, e_{j}\right\rangle\right)_{i, j}$ of the vectors $e_{i}, i \in I$, associated to $P$ is a symmetric matrix of signature $(1,3)$, whose rank is four. In general, for an $n$-dimensional non-Euclidean polyhedron, the Gram matrix has rank at most $n+1$. For an $n$-dimensional Euclidean polyhedron, the Gram matrix has rank at most $n$ (see [1]).

Also $\left\langle e_{i}, e_{j}\right\rangle$ has the following geometrical meaning (see [9]):

$$
\left\langle e_{i}, e_{j}\right\rangle= \begin{cases}-\cos \angle\left(e_{i}^{\perp}, e_{j}^{\perp}\right) & \text { if } e_{i}^{\perp} \text { and } e_{j}^{\perp} \text { intersect, } \\ -1 & \text { if } e_{i}^{\perp} \text { and } e_{j}^{\perp} \text { are parallel, } \\ -\cosh d\left(e_{i}^{\perp}, e_{j}^{\perp}\right) & \text { if } e_{i}^{\perp} \text { and } e_{j}^{\perp} \text { are ultra parallel, }\end{cases}
$$

where $\angle\left(e_{i}^{\perp}, e_{j}^{\perp}\right)$ denotes the angle between $e_{i}^{\perp}$ and $e_{j}^{\perp}$, and $d\left(e_{i}^{\perp}, e_{j}^{\perp}\right)$ denotes the distance between $e_{i}^{\perp}$ and $e_{j}^{\perp}$.

\section{Some Known Volume Formulas of Hyperbolic Polyhedra}

\subsection{Volume of Orthoschemes}

In the Euclidean, hyperbolic, and spherical space, orthoschemes are the most basic objects in polyhedral geometry, since an arbitrary polyhedron can be represented as an 


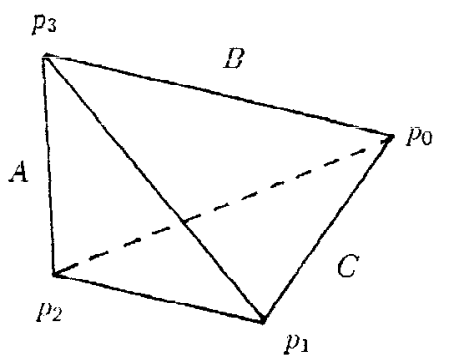

Fig. 3.1

algebraic sum of orthoschemes. In general, an $n$-orthoscheme is a bounded $n$-simplex with vertices $p_{0}, \ldots, p_{n}$ such that

$$
\operatorname{span}\left(p_{0}, \ldots, p_{i}\right) \perp \operatorname{span}\left(p_{i}, \ldots, p_{n}\right),
$$

for $i=1, \ldots, n-1$. In the three-dimensional case we get an orthoscheme $p_{0} p_{1} p_{2} p_{3}$ such that the edge $p_{0} p_{1}$ is orthogonal to the plane $p_{1} p_{2} p_{3}$ and the plane $p_{0} p_{1} p_{2}$ is orthogonal to the edge $p_{2} p_{3}$.

Of the six dihedral angles of the orthoscheme, three are right angles. We denote the other three by $A, B, C$ as in Fig. 3.1. Let $O T(A, B, C)$ be the orthoscheme with the dihedral angles $A, B, C$, and let $a, b, c$ denote the lengths of the corresponding edges of dihedral angles $A, B, C$, respectively. The Gram matrix of $O T(A, B, C)$ is of the form

$$
\left(\begin{array}{cccc}
1 & -\cos A & 0 & 0 \\
-\cos A & 1 & -\cos B & 0 \\
0 & -\cos B & 1 & -\cos C \\
0 & 0 & -\cos C & 1
\end{array}\right)
$$

and the determinant is given by

$$
\Delta=\sin ^{2} A \sin ^{2} C-\cos ^{2} B .
$$

We know that $\Delta$ is negative since the signature is $(1,3)$.

We can obtain the following facts from [11]. For the existence of an orthoscheme in $\mathbb{H}^{3}$ with given dihedral angles $A, B, C \in(0, \pi)$, it is necessary and sufficient that the determinant $\Delta$ is negative and $A, B, C$ are acute, and

$$
A+B \geq \frac{\pi}{2}, \quad C+B \geq \frac{\pi}{2},
$$

where one equality holds whenever one of the vertices $p_{3}, p_{0}$ is a point at infinity of $\mathbb{H}^{3}$. We also have another important property:

$$
\tan A \tanh a=\tan \left(\frac{\pi}{2}-B\right) \tanh b=\tan C \tanh c=\frac{\sqrt{-\Delta}}{\cos A \cos C} .
$$


Usually, one defines an additional (acute) angle $\delta$ by the equation

$$
\tan \delta:=\frac{\sqrt{-\Delta}}{\cos A \cos C}
$$

and we have $\delta \leq \min \{A, C, \pi / 2-B\}$.

Remark. There are similar facts about the orthoscheme in the spherical space which are due to Schläfli (see [2] and [5]).

The volume of the orthoschemes can be calculated by the following formula which is essentially due to Lobachevsky (see [11] or [1]):

$$
\begin{aligned}
\operatorname{vol} O T(A, B, C)=\frac{1}{4}[ & \wedge(A+\delta)-\wedge(A-\delta)+\wedge(C+\delta)-\wedge(C-\delta) \\
& \left.-\wedge\left(\frac{\pi}{2}-B+\delta\right)+\wedge\left(\frac{\pi}{2}-B-\delta\right)+2 \wedge\left(\frac{\pi}{2}-\delta\right)\right],
\end{aligned}
$$

where

$$
0 \leq \delta=\arctan \frac{\sqrt{-\Delta}}{\cos A \cos C}<\frac{\pi}{2}
$$

\subsection{Volumes of Ideal Tetrahedra and Ideal Polyhedra}

We now consider a tetrahedron $T$, all of whose vertices are at infinity. In this case, these vertices are called ideal vertices and the tetrahedron is called an ideal tetrahedron.

Let $T\left(A, B, C, A^{\prime}, B^{\prime}, C^{\prime}\right)$ be a tetrahedron with dihedral angles $A, B, C$ and $A^{\prime}, B^{\prime}$, $C^{\prime}$, where $A$ and $A^{\prime}$ are dihedral angles at opposite edges and also for $B, B^{\prime}$ and $C, C^{\prime}$. The sum of the dihedral angles of a tetrahedron at any vertex at infinity is the sum of the angles of the Euclidean triangle cut by a sufficiently small horosphere with center at this vertex, and hence is equal to $\pi$. So, for the ideal tetrahedron $T\left(A, B, C, A^{\prime}, B^{\prime}, C^{\prime}\right)$ we obtain four equalities and we deduce that $A=A^{\prime}, B=B^{\prime}, C=C^{\prime}$, and $A+B+C=\pi$.

The volume of an ideal tetrahedron $I T(A, B, C)$ can be calculated by means of a partition into six orthoschemes by dropping the perpendicular from one vertex onto the plane of the opposite face and onto the lines bounding this face from the foot of the perpendicular (Fig. 3.2). Then we get six orthoschemes every two of which have dihedral angles $(A, \pi / 2-A, A),(B, \pi / 2-B, B)$, or $(C, \pi / 2-C, C)$, and the volumes of the six orthoschemes are easily obtained by formula $(7)$.

Therefore we have

$$
\begin{aligned}
\operatorname{vol} I T(A, B, C)= & 2 \operatorname{vol} O T\left(A, \frac{\pi}{2}-A, A\right)+2 \operatorname{vol} O T\left(B, \frac{\pi}{2}-B, B\right) \\
& +2 \operatorname{vol} O T\left(C, \frac{\pi}{2}-C, C\right) \\
= & \wedge(A)+\wedge(B)+\wedge(C) .
\end{aligned}
$$

This formula was first observed by Milnor [7]. In his paper he showed this formula by using a method of direct integration instead of using the volume formula of an orthoscheme. 


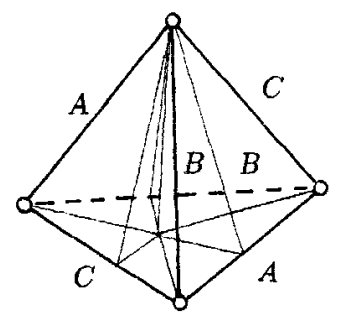

Fig. 3.2

There is a fundamental result on hyperbolic ideal polyhedra [8] which says that convex hyperbolic ideal polyhedra are uniquely determined by their dihedral angles. From the Milnor formula, one can deduce the volume formulas of various types of ideal polyhedra such as ideal cube, ideal pyramid, ideal regular prism, ideal regular antiprism, etc., into simple forms (see [12]). However, even for the cases of ideal octahedron, ideal dodecahedron, or ideal icosahedron, what the volume formulas of these polyhedra are, does not seem to be known. The volume formulas for ideal octahedra and others are given in the thesis of the first author.

The volume of tetrahedra $I T$ with one ideal vertex is obtained from the volume of $n$-pyramids with apex at infinity with condition $n=3$ (see [1] or [10]). Then the volume formula of tetrahedra with one ideal vertex is easily changed to a nicer and more symmetric formula as follows:

$\operatorname{vol} \operatorname{IT}\left(A, B, C, A^{\prime}, B^{\prime}, C^{\prime}\right)$

$$
\begin{aligned}
=\frac{1}{2}[ & \wedge\left(\frac{A-B-C+\pi}{2}\right)+\wedge\left(\frac{-A+B-C+\pi}{2}\right)+\wedge\left(\frac{-A-B+C+\pi}{2}\right) \\
& -\wedge\left(\frac{A+B+C+\pi}{2}\right)+\wedge\left(\frac{A-B^{\prime}-C^{\prime}+\pi}{2}\right) \\
& +\wedge\left(\frac{-A+B^{\prime}-C^{\prime}+\pi}{2}\right)+\wedge\left(\frac{-A-B^{\prime}+C^{\prime}+\pi}{2}\right) \\
& -\wedge\left(\frac{A+B^{\prime}+C^{\prime}+\pi}{2}\right)+\wedge\left(\frac{A^{\prime}-B-C^{\prime}+\pi}{2}\right) \\
& +\wedge\left(\frac{-A^{\prime}+B-C^{\prime}+\pi}{2}\right)+\wedge\left(\frac{-A^{\prime}-B+C^{\prime}+\pi}{2}\right) \\
& -\wedge\left(\frac{A^{\prime}+B+C^{\prime}+\pi}{2}\right)+\wedge\left(\frac{A^{\prime}-B^{\prime}-C+\pi}{2}\right) \\
& +\wedge\left(\frac{-A^{\prime}+B^{\prime}-C+\pi}{2}\right)+\wedge\left(\frac{-A^{\prime}-B^{\prime}+C+\pi}{2}\right) \\
& \left.-\wedge\left(\frac{A^{\prime}+B^{\prime}+C+\pi}{2}\right)\right]
\end{aligned}
$$




$$
\begin{aligned}
& +\wedge\left(\frac{A+A^{\prime}+B+B^{\prime}}{2}\right)+\wedge\left(\frac{A+A^{\prime}+C+C^{\prime}}{2}\right) \\
& +\wedge\left(\frac{B+B^{\prime}+C+C^{\prime}}{2}\right) .
\end{aligned}
$$

It is interesting to notice that the above formula does not show any preference of choice of the ideal vertex.

\section{A Volume Formula for Arbitrary Hyperbolic Tetrahedra}

We can obtain the volume formula for arbitrary compact tetrahedra $T$ by means of extending each edge to infinity (Fig. 4.1) and making four ideal polyhedra $\mathrm{H}_{1}, \mathrm{H}_{2}, \mathrm{H}_{3}$, and $H_{4}$ (Figs. 4.2-4.5).

Extend to infinity the six edges near vertices $p_{0}$ and $p_{1}$ outward from the tetrahedron $T$, and let the ideal points be $1,1^{\prime}, 2,3,4$, and 5 . Then we obtain the planes $\left(1,2,3,1^{\prime}\right)$, $\left(1,4,5,1^{\prime}\right),(1,2,4),\left(1^{\prime}, 3,5\right),(2,3,5)$, and $(2,4,5)$. We denote the resulting ideal polyhedron as $H_{1}$. The ideal polyhedron $H_{1}$ is an ideal hexahedron and becomes an ideal prism if the planes $(2,3,5)$ and $(2,4,5)$ coincide to form a single plane $(2,3,5,4)$ (i.e., if the dihedral angle of the edge $(2,5)$ is $\pi$ ). If the dihedral angle of edge $(2,5)$ is smaller (resp. larger) than $\pi$, then the ideal hexahedron $H_{1}$ becomes a convex (resp. not-convex) polyhedron. The ideal polyhedra $\mathrm{H}_{2}, \mathrm{H}_{3}$, and $\mathrm{H}_{4}$ are constructed similarly.

Each of the ideal polyhedra $\mathrm{H}_{1}, \mathrm{H}_{2}, \mathrm{H}_{3}$, and $\mathrm{H}_{4}$ can be divided algebraically into smaller pieces as follows. The ideal polyhedron $H_{1}$ is decomposed as a tetrahedron $\left(p_{1}, 1,2,4\right)$ plus a tetrahedron $\left(p_{0}, 1^{\prime}, 3,5\right)$ plus a polyhedron $\left(p_{0}, p_{1}, 2,4,5,3\right)$, and the ideal polyhedron $H_{2}$ is decomposed as a tetrahedron $\left(p_{3}, 4,5,6\right)$ plus a tetrahedron $\left(p_{2}, 2,3,6^{\prime}\right)$ plus a polyhedron $\left(p_{0}, p_{1}, 2,4,5,3\right)$ minus a tetrahedron $\left(p_{0}, p_{1}, p_{2}, p_{3}\right)$ (Figs. 4.2 and 4.3). Similarly the ideal polyhedron $H_{3}$ is decomposed as a tetrahedron $\left(p_{2}, 2^{\prime}, 3^{\prime}, 6\right)$ plus a tetrahedron $\left(p_{3}, 4^{\prime}, 5^{\prime}, 6^{\prime}\right)$ plus a polyhedron $\left(p_{3}, p_{2}, 2^{\prime}, 3^{\prime}, 5^{\prime}, 4^{\prime}\right)$, and the ideal polyhedron $H_{4}$ is decomposed as a tetrahedron $\left(p_{0}, 1,3^{\prime}, 5^{\prime}\right)$ plus a tetrahedron $\left(p_{1}, 1^{\prime}, 2^{\prime}, 4^{\prime}\right)$ plus a polyhedron $\left(p_{3}, p_{2}, 2^{\prime}, 3^{\prime}, 5^{\prime}, 4^{\prime}\right)$ minus a tetrahedron $\left(p_{0}, p_{1}, p_{2}, p_{3}\right)$ (Figs. 4.4 and 4.5).

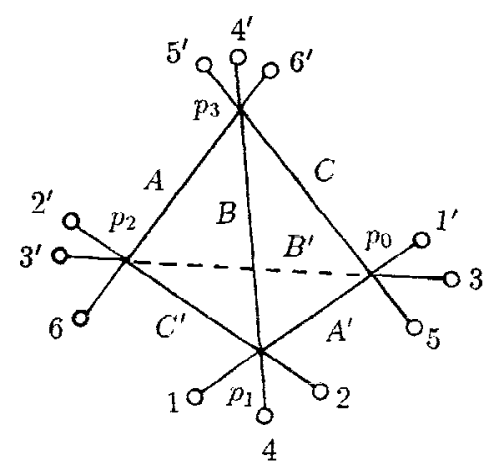

Fig. 4.1 


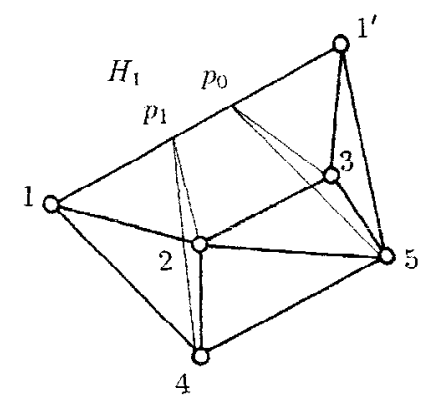

Fig. 4.2

So we have the following relation:

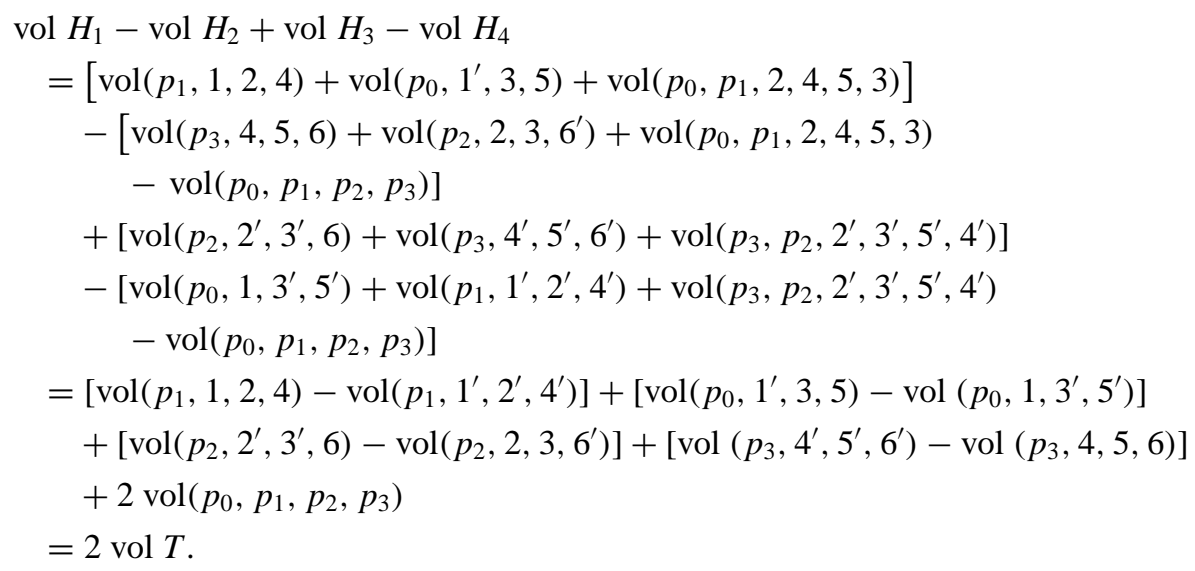

Here two tetrahedra $\left(p_{1}, 1,2,4\right)$ and $\left(p_{1}, 1^{\prime}, 2^{\prime}, 4^{\prime}\right)$ are symmetric with respect to the point $p_{1}$ and hence they are isometric and have the same volume. Similarly, other pairs are also symmetric with respect to the vertices of the given original tetrahedron $T$. So the last equality follows.

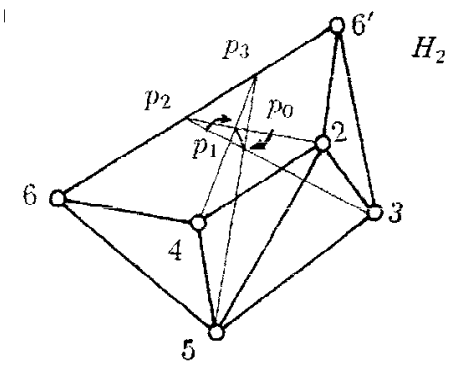

Fig. 4.3 


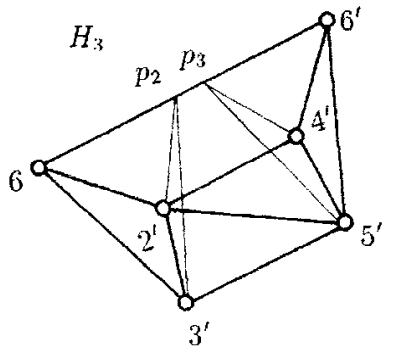

Fig. 4.4

The ideal polyhedron of combinatorial type $H_{i}$ is divided into three ideal tetrahedra (Fig. 4.6), so we can obtain the volume formula of $H_{i}$ represented by its dihedral angles, even though $H_{i}$ is not convex.

By the Milnor formula, the volume of ideal polyhedron $H_{i}$ is represented by

$$
\begin{aligned}
\operatorname{vol} H_{i}= & {\left[\wedge\left(\beta_{1}\right)+\wedge\left(\beta_{2}\right)+\wedge\left(\beta_{3}\right)\right]+\left[\wedge\left(\alpha_{1}\right)+\wedge\left(\pi-\beta_{3}\right)+\wedge\left(\alpha_{4}-\beta_{2}\right)\right] } \\
& +\left[\wedge\left(\alpha_{2}\right)+\wedge\left(\alpha_{3}\right)+\wedge\left(\gamma_{2}\right)\right] \\
= & \left.\wedge\left(\beta_{1}\right)+\wedge\left(\beta_{2}\right)+\wedge\left(\alpha_{1}\right)+\wedge\left(\alpha_{4}-\beta_{2}\right)+\wedge\left(\alpha_{2}\right)+\wedge\left(\alpha_{3}\right)+\wedge\left(\gamma_{2}\right)\right]
\end{aligned}
$$

In the components of an ideal polyhedron $H_{i}$, the dihedral angles of the subdividing tetrahedra are determined by the dihedral angles of the original tetrahedron $T$ and the angle conditions around the ideal vertices. However, the dihedral angles of the polyhedra $\left(p_{0}, p_{1}, 2,4,5,3\right)$ and $\left(p_{3}, p_{2}, 2^{\prime}, 3^{\prime}, 5^{\prime}, 4^{\prime}\right)$ are not completely determined from the dihedral angles of the original tetrahedron $T$. So we let the undetermined angles of these polyhedra be $a, b, c, d$ and $a^{\prime}, b^{\prime}, c^{\prime}, d^{\prime}$, respectively, as shown in Figs. 4.7 and 4.8. From the angle conditions around the ideal vertices, we have the following equations:

$$
\begin{aligned}
& a+b=B, \quad c+d=B^{\prime}, \quad b+c+e=C^{\prime}, \quad a+d+e=C, \\
& a^{\prime}+b^{\prime}=B, \quad c^{\prime}+d^{\prime}=B^{\prime}, \quad b^{\prime}+c^{\prime}+e^{\prime}=C^{\prime}, \quad a^{\prime}+d^{\prime}+e^{\prime}=C \text {. }
\end{aligned}
$$

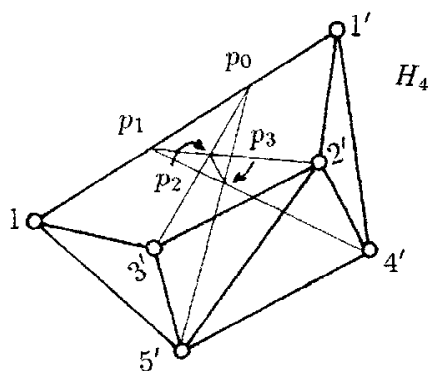

Fig. 4.5 
$H_{i}$
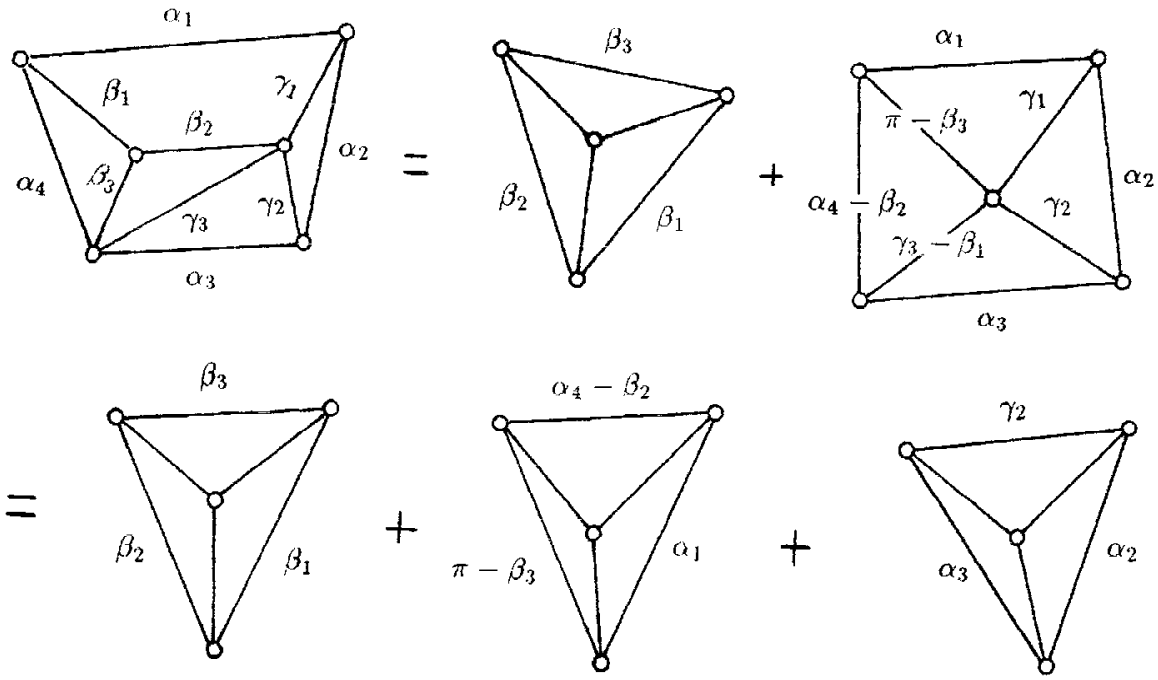

Fig. 4.6

Equations (11) are easily changed into

$$
\begin{array}{cc}
a+b=B, & b+c=\left(B+B^{\prime}-C+C^{\prime}\right) / 2, \quad e=\left(C+C^{\prime}-B-B^{\prime}+2 \pi\right) / 2, \\
c+d=B^{\prime}, \quad a+d=\left(B+B^{\prime}+C-C^{\prime}\right) / 2, \\
a^{\prime}+b^{\prime}=B, \quad b^{\prime}+c^{\prime}=\left(B+B^{\prime}-C+C^{\prime}\right) / 2, \quad e^{\prime}=\left(C+C^{\prime}-B-B^{\prime}+2 \pi\right) / 2, \\
c^{\prime}+d^{\prime}=B^{\prime}, \quad a^{\prime}+d^{\prime}=\left(B+B^{\prime}+C-C^{\prime}\right) / 2 .
\end{array}
$$

From (11), we know that each polyhedron $\left(p_{0}, p_{1}, 2,4,5,3\right)$ and $\left(p_{3}, p_{2}, 2^{\prime}, 3^{\prime}, 5^{\prime}, 4^{\prime}\right)$ has one unknown variable. To determine the unknown variable, we need one more condition about the dihedral angles, which will follow from the Gram matrix later. From the ideal vertices conditions and (10), we can deduce a volume formula for $H_{1}$ and $H_{2}$. See Figs. 4.9 and 4.10 for the dihedral angles of $H_{1}$ and $H_{2}$.

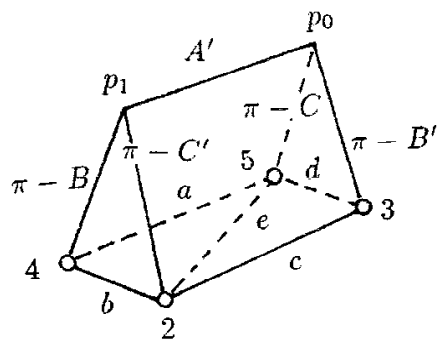

Fig. 4.7 


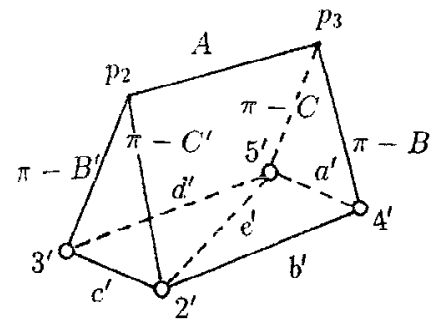

Fig. 4.8

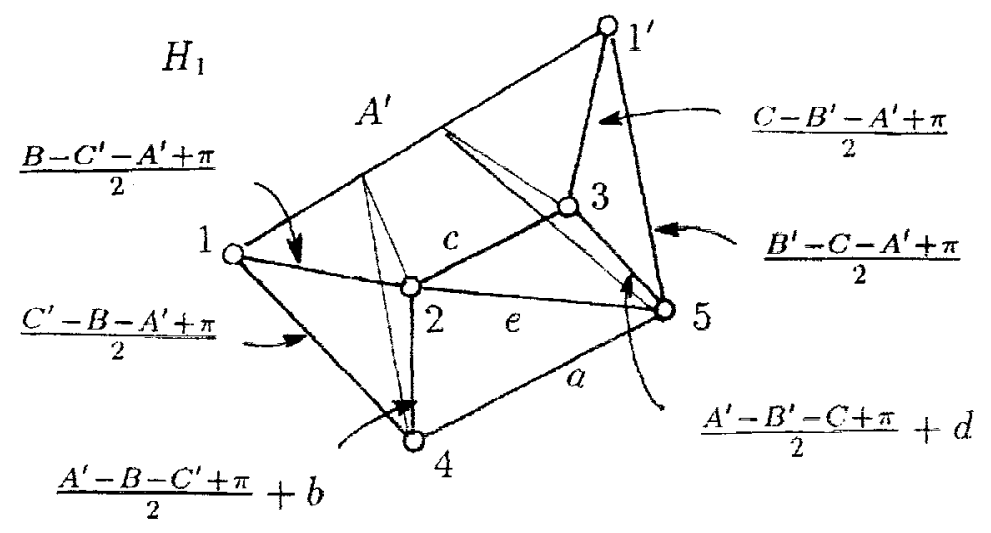

Fig. 4.9

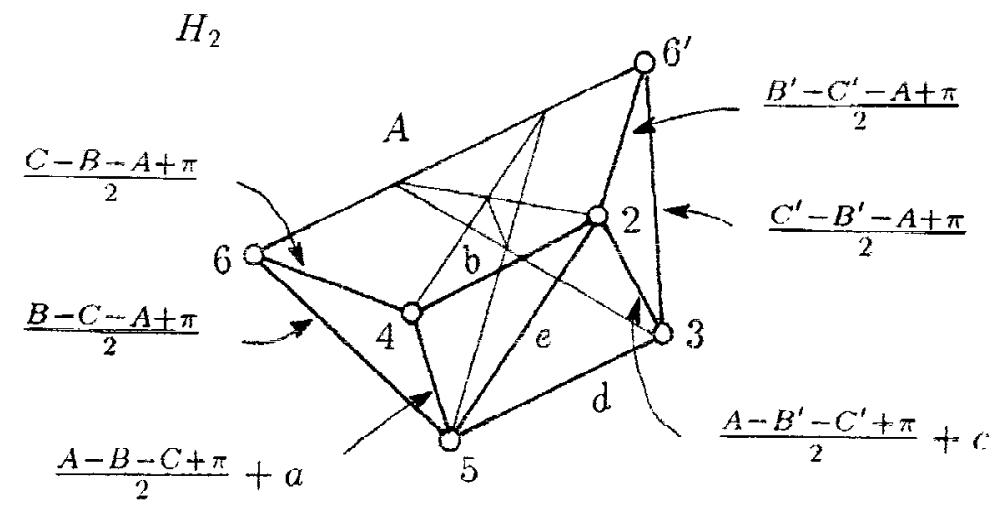

Fig. 4.10 
We get

$$
\begin{aligned}
\operatorname{vol} H_{1}= & \wedge\left(A^{\prime}\right)+\wedge\left(\frac{C^{\prime}-B-A^{\prime}+\pi}{2}\right)+\wedge(a)+\wedge\left(\frac{B^{\prime}-C-A^{\prime}+\pi}{2}-c\right) \\
& +\wedge\left(\frac{C-B^{\prime}-A^{\prime}+\pi}{2}\right)+\wedge(c)+\wedge\left(\frac{A^{\prime}-B-C^{\prime}+\pi}{2}+b\right), \\
\operatorname{vol} H_{2}= & \wedge(A)+\wedge\left(\frac{C^{\prime}-B^{\prime}-A+\pi}{2}\right)+\wedge(d)+\wedge\left(\frac{B-C-A+\pi}{2}-b\right) \\
& +\wedge(b)+\wedge\left(\frac{C-B-A+\pi}{2}\right)+\wedge\left(\frac{A-B^{\prime}-C^{\prime}+\pi}{2}+c\right) .
\end{aligned}
$$

Therefore we have

vol $H_{1}-\operatorname{vol} H_{2}$

$$
\begin{aligned}
= & \wedge\left(A^{\prime}\right)-\wedge(A)+\wedge\left(\frac{C^{\prime}-B-A^{\prime}+\pi}{2}\right)-\wedge\left(\frac{C^{\prime}-B^{\prime}-A+\pi}{2}\right) \\
& +\wedge\left(\frac{C-B^{\prime}-A^{\prime}+\pi}{2}\right)-\wedge\left(\frac{C-B-A+\pi}{2}\right) \\
& +\wedge(a)-\wedge(b)+\wedge(c)-\wedge(d) \\
& +\wedge\left(\frac{B^{\prime}-C-A^{\prime}+\pi}{2}-c\right)+\wedge\left(\frac{A^{\prime}-B-C^{\prime}+\pi}{2}+b\right) \\
& -\wedge\left(\frac{B-C-A+\pi}{2}-b\right)-\wedge\left(\frac{A-B^{\prime}-C^{\prime}+\pi}{2}+c\right),
\end{aligned}
$$

and similarly we have

$$
\begin{aligned}
& \operatorname{vol} \mathrm{H}_{3}-\operatorname{vol} \mathrm{H}_{4} \\
& =\wedge(A)-\wedge\left(A^{\prime}\right)+\wedge\left(\frac{C-B-A+\pi}{2}\right)-\wedge\left(\frac{C-B^{\prime}-A^{\prime}+\pi}{2}\right) \\
& +\wedge\left(\frac{C^{\prime}-B^{\prime}-A+\pi}{2}\right)-\wedge\left(\frac{C^{\prime}-B-A^{\prime}+\pi}{2}\right) \\
& -\wedge\left(a^{\prime}\right)+\wedge\left(b^{\prime}\right)-\wedge\left(c^{\prime}\right)+\wedge\left(d^{\prime}\right) \\
& +\wedge\left(\frac{B-C-A+\pi}{2}-b^{\prime}\right)+\wedge\left(\frac{A-B^{\prime}-C^{\prime}+\pi}{2}+c^{\prime}\right) \\
& -\wedge\left(\frac{B^{\prime}-C-A^{\prime}+\pi}{2}-c^{\prime}\right)-\wedge\left(\frac{A^{\prime}-B-C^{\prime}+\pi}{2}+b^{\prime}\right) .
\end{aligned}
$$

Consequently,

$$
\begin{aligned}
2 \operatorname{vol} T= & \operatorname{vol} H_{1}-\operatorname{vol} H_{2}+\operatorname{vol} H_{3}-\operatorname{vol} H_{4} \\
= & \wedge(a)-\wedge(b)+\wedge(c)-\wedge(d)+\wedge\left(\frac{B^{\prime}-C-A^{\prime}+\pi}{2}-c\right) \\
& +\wedge\left(\frac{A^{\prime}-B-C^{\prime}+\pi}{2}+b\right)-\wedge\left(\frac{B-C-A+\pi}{2}-b\right)
\end{aligned}
$$




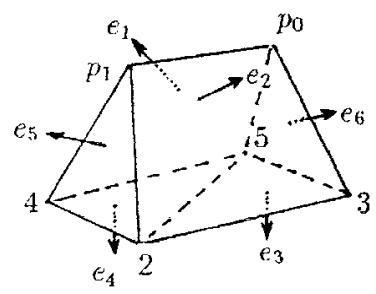

Fig. 4.11

$$
\begin{aligned}
& -\wedge\left(\frac{A-B^{\prime}-C^{\prime}+\pi}{2}+c\right)-\wedge\left(a^{\prime}\right)+\wedge\left(b^{\prime}\right)-\wedge\left(c^{\prime}\right)+\wedge\left(d^{\prime}\right) \\
& +\wedge\left(\frac{B-C-A+\pi}{2}-b^{\prime}\right)+\wedge\left(\frac{A-B^{\prime}-C^{\prime}+\pi}{2}+c^{\prime}\right) \\
& -\wedge\left(\frac{B^{\prime}-C-A^{\prime}+\pi}{2}-c^{\prime}\right)-\wedge\left(\frac{A^{\prime}-B-C^{\prime}+\pi}{2}+b^{\prime}\right) .
\end{aligned}
$$

We suppose that the angle sum of $C$ and $C^{\prime}$ is larger than the angle sum of $B$ and $B^{\prime}$, then the polyhedra $\left(p_{0}, p_{1}, 2,4,5,3\right)$ and $\left(p_{3}, p_{2}, 2^{\prime}, 3^{\prime}, 5^{\prime}, 4^{\prime}\right)$ become convex polyhedra. Now we number the faces of $\left(p_{0}, p_{1}, 2,4,5,3\right)$ and $\left(p_{3}, p_{2}, 2^{\prime}, 3^{\prime}, 5^{\prime}, 4^{\prime}\right)$ as shown in Figs. 4.11 and 4.12.

The order of the numbering of each polyhedron is not important. However, the numbering of one polyhedron is automatically determined from the numbering of the other, and the directions of the outward unit normal vectors are opposite to the directions of the former polyhedron (see Fig. 4.13).

One unknown variable among the dihedral angles of the polyhedron $\left(p_{0}, p_{1}, 2,4,5,3\right)$ is determined by the following Gram matrix $\left(\left\langle e_{i}, e_{j}\right\rangle\right)$ :

$G=\left(\begin{array}{cccccc}1 & -\cos A^{\prime} & \cos (a+e) & -\cos a & \cos B & \cos C \\ -\cos A^{\prime} & 1 & -\cos c & \cos (c+e) & \cos C^{\prime} & \cos B^{\prime} \\ \cos (a+e) & -\cos c & 1 & -\cos e & \cos (b+e) & -\cos d \\ -\cos a & \cos (c+e) & -\cos e & 1 & -\cos b & \cos (d+e) \\ \cos B & \cos C^{\prime} & \cos (b+e) & -\cos b & 1 & -\cos A \\ \cos C & \cos B^{\prime} & -\cos d & \cos (d+e) & -\cos A & 1\end{array}\right)$.

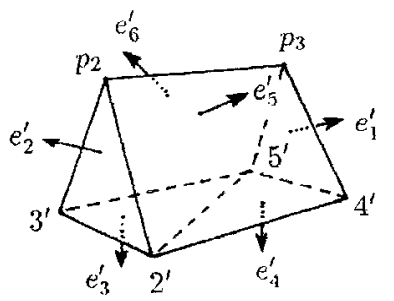

Fig. 4.12 


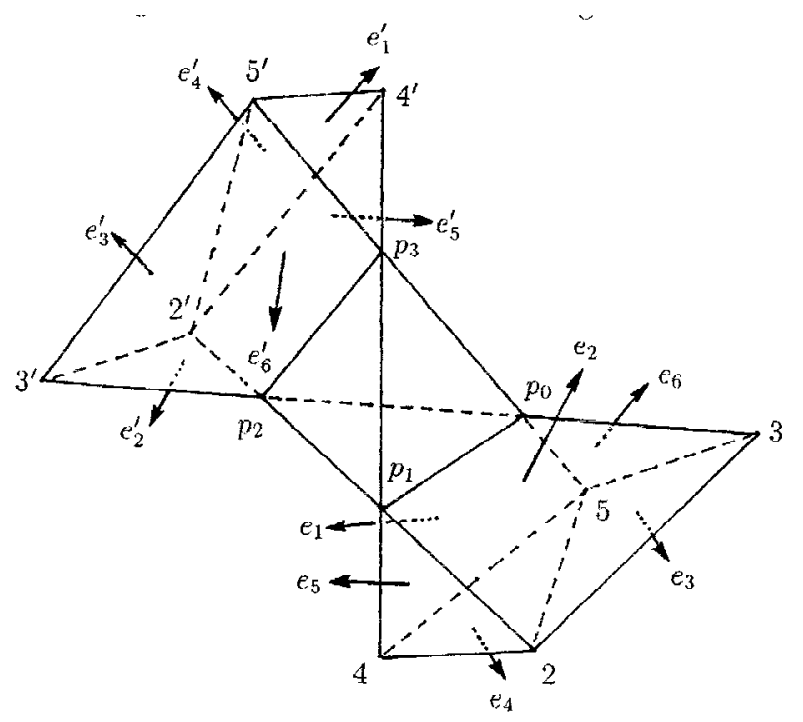

Fig. 4.13

Here the $(1,3),(2,4),(3,5)$, and $(4,6)$ components of matrix $G$ are calculated using the Euclidean quadrilaterals cut by small horospheres with centers at the two ideal vertices 2 and 5.

The Gram matrix of $\left(p_{3}, p_{2}, 2^{\prime}, 3^{\prime}, 5^{\prime}, 4^{\prime}\right)$ can be obtained from the matrix given in (14) by replacing $a, b, c$, and $d$ with $a^{\prime}, b^{\prime}, c^{\prime}$, and $d^{\prime}$, respectively. Hence the corresponding two unknown variables of the dihedral angles of $\left(p_{0}, p_{1}, 2,4,5,3\right)$ and $\left(p_{3}, p_{2}, 2^{\prime}, 3^{\prime}\right.$, $\left.5^{\prime}, 4^{\prime}\right)$ are two roots of the same equation.

From the rank condition of the Gram matrix, we eliminate the third row and third column (or any suitable choice) and take the determinant to be zero, then we can obtain the volume formula of a tetrahedron.

We restricted the angle condition so that the angle sum of $C$ and $C^{\prime}$ is larger than the angle sum of $B$ and $B^{\prime}$. However, we do not need this angle condition. Although these polyhedra $\left(p_{0}, p_{1}, 2,4,5,3\right)$ and $\left(p_{3}, p_{2}, 2^{\prime}, 3^{\prime}, 5^{\prime}, 4^{\prime}\right)$ are not convex, the Gram matrix of the two polyhedra also have the same representation (14) in this case and the rank condition of the Gram matrix is also satisfied in any polyhedron. So the above formula can be adapted for arbitrary tetrahedra.

Theorem 1. The volume formula of tetrahedra $T\left(A, B, C, A^{\prime}, B^{\prime}, C^{\prime}\right)$ is represented by

$$
\begin{aligned}
2 \operatorname{vol} T= & \wedge(a)-\wedge(b)+\wedge(c)-\wedge(d)-\wedge\left(\frac{B-C-A+\pi}{2}-b\right) \\
& +\wedge\left(\frac{A^{\prime}-B-C^{\prime}+\pi}{2}+b\right)+\wedge\left(\frac{B^{\prime}-C-A^{\prime}+\pi}{2}-c\right)
\end{aligned}
$$




$$
\begin{aligned}
& -\wedge\left(\frac{A-B^{\prime}-C^{\prime}+\pi}{2}+c\right) \\
& -\wedge\left(a^{\prime}\right)+\wedge\left(b^{\prime}\right)-\wedge\left(c^{\prime}\right)+\wedge\left(d^{\prime}\right)+\wedge\left(\frac{B-C-A+\pi}{2}-b^{\prime}\right) \\
& -\wedge\left(\frac{A^{\prime}-B-C^{\prime}+\pi}{2}+b^{\prime}\right)-\wedge\left(\frac{B^{\prime}-C-A^{\prime}+\pi}{2}-c^{\prime}\right) \\
& +\wedge\left(\frac{A-B^{\prime}-C^{\prime}+\pi}{2}+c^{\prime}\right),
\end{aligned}
$$

where the values $a, b, c, d, a^{\prime}, b^{\prime}, c^{\prime}, d^{\prime}$ are determined by the equations

$$
\begin{aligned}
& a+b=B, \quad c+d=B^{\prime}, \quad b+c+e=C^{\prime}, \quad a+d+e=C, \\
& a^{\prime}+b^{\prime}=B, \quad c^{\prime}+d^{\prime}=B^{\prime}, \quad b^{\prime}+c^{\prime}+e^{\prime}=C^{\prime}, \quad a^{\prime}+d^{\prime}+e^{\prime}=C,
\end{aligned}
$$

and

$$
G_{1}=\left|\begin{array}{ccccc}
1 & -\cos A^{\prime} & -\cos a & \cos B & \cos C \\
-\cos A^{\prime} & 1 & \cos (c+e) & \cos C^{\prime} & \cos B^{\prime} \\
-\cos a & \cos (c+e) & 1 & -\cos b & \cos (d+e) \\
\cos B & \cos C^{\prime} & -\cos b & 1 & -\cos A \\
\cos C & \cos B^{\prime} & \cos (d+e) & -\cos A & 1
\end{array}\right|=0
$$

and

$$
G_{2}=\left|\begin{array}{ccccc}
1 & -\cos A^{\prime} & -\cos a^{\prime} & \cos B & \cos C \\
-\cos A^{\prime} & 1 & \cos \left(c^{\prime}+e^{\prime}\right) & \cos C^{\prime} & \cos B^{\prime} \\
-\cos a^{\prime} & \cos \left(c^{\prime}+e^{\prime}\right) & 1 & -\cos b^{\prime} & \cos \left(d^{\prime}+e^{\prime}\right) \\
\cos B & \cos C^{\prime} & -\cos b^{\prime} & 1 & -\cos A \\
\cos C & \cos B^{\prime} & \cos \left(d^{\prime}+e^{\prime}\right) & -\cos A & 1
\end{array}\right|=0
$$

The following examples are applications of the above theorem to special tetrahedra.

Example 1. What is the volume of $T\left(A, B, C, A, B^{\prime}, C\right)$ ? Formulas (12) and (13) induce the following volume formula:

$$
\begin{aligned}
2 \operatorname{vol} T(A, B, & \left.C, A, B^{\prime}, C\right) \\
= & \wedge(a)-\wedge(b)+\wedge(c)-\wedge(d) \\
& -\wedge\left(\frac{B-C-A+\pi}{2}-b\right)+\wedge\left(\frac{A-B-C+\pi}{2}+b\right) \\
& +\wedge\left(\frac{B^{\prime}-C-A+\pi}{2}-c\right)-\wedge\left(\frac{A-B^{\prime}-C+\pi}{2}+c\right) \\
& -\wedge\left(a^{\prime}\right)+\wedge\left(b^{\prime}\right)-\wedge\left(c^{\prime}\right)+\wedge\left(d^{\prime}\right) \\
& +\wedge\left(\frac{B-C-A+\pi}{2}-b^{\prime}\right)-\wedge\left(\frac{A-B-C+\pi}{2}+b^{\prime}\right) \\
& -\wedge\left(\frac{B^{\prime}-C-A+\pi}{2}-c^{\prime}\right)+\wedge\left(\frac{A-B^{\prime}-C+\pi}{2}+c^{\prime}\right),
\end{aligned}
$$


where $a+b=B, c+d=B^{\prime}$, and $b+c=\left(B+B^{\prime}\right) / 2=a+d$. Here the undetermined variable $a$ and $a^{\prime}$ are calculated from the fact that the cofactors $G_{1}$ and $G_{2}$ of matrix $G$ are zero. From the equation $G_{1}=0$, it follows through a long calculation (in fact I used the computer program Mathematica for the following factorization) that

$$
\begin{gathered}
M\left(\cos B+\cos B^{\prime}+2 \cos A \cos C\right)(\cos B \cos 2 a+\sin B \sin 2 a) \\
=M\left(1+\cos B \cos B^{\prime}-\cos ^{2} A-\cos ^{2} C\right),
\end{gathered}
$$

where

$$
\begin{aligned}
M= & 1+\cos ^{2} A-\cos ^{2} B+2 \cos A \cos B \cos C-\cos ^{2} C+2 \cos ^{2} B \cos ^{2} C \\
& +2 \cos A \sin B \sin C+2 \cos B \sin B \cos C \sin C .
\end{aligned}
$$

Therefore we have

$$
\begin{aligned}
& \cos (2 a-B)=\frac{1+\cos B \cos B^{\prime}-\cos ^{2} A-\cos ^{2} C}{\cos B+\cos B^{\prime}+2 \cos A \cos C}=: \cos w, \\
& \cos \left(2 a^{\prime}-B\right)=\frac{1+\cos B \cos B^{\prime}-\cos ^{2} A-\cos ^{2} C}{\cos B+\cos B^{\prime}+2 \cos A \cos C}=: \cos w,
\end{aligned}
$$

and we can write $a=(B+w) / 2$ or $(B-w) / 2$. If two angles $a$ and $a^{\prime}$ have the same value, the volume of $T\left(A, B, C, A, B^{\prime}, C\right)$ vanishes. So we have $a=(B+w) / 2$, $a^{\prime}=(B-w) / 2$ or $a=(B-w) / 2, a^{\prime}=(B+w) / 2$. Here we choose $a=(B+w) / 2$. Then we have

$$
\begin{aligned}
(a, b, c, d) & =\left(\frac{B+w}{2}, \frac{B-w}{2}, \frac{B^{\prime}+C^{\prime}-C+w}{2}, \frac{B^{\prime}+C-C^{\prime}-w}{2}\right) \\
& =\left(\frac{B+w}{2}, \frac{B-w}{2}, \frac{B^{\prime}+w}{2}, \frac{B^{\prime}-w}{2}\right)
\end{aligned}
$$

and

$$
\begin{aligned}
\left(a^{\prime}, b^{\prime}, c^{\prime}, d^{\prime}\right) & =\left(\frac{B-w}{2}, \frac{B+w}{2}, \frac{B^{\prime}+C^{\prime}-C-w}{2}, \frac{B^{\prime}+C-C^{\prime}-w}{2}\right) \\
& =\left(\frac{B-w}{2}, \frac{B+w}{2}, \frac{B^{\prime}-w}{2}, \frac{B^{\prime}+w}{2}\right) .
\end{aligned}
$$

Therefore we get

$$
\begin{aligned}
\operatorname{vol} T(A, B, C & \left., A, B^{\prime}, C\right) \\
= & \wedge\left(\frac{B+w}{2}\right)-\wedge\left(\frac{B-w}{2}\right)+\wedge\left(\frac{B^{\prime}+w}{2}\right)-\wedge\left(\frac{B^{\prime}-w}{2}\right) \\
& -\wedge\left(\frac{A+C+\pi+w}{2}\right)+\wedge\left(\frac{A+C+\pi-w}{2}\right) \\
& -\wedge\left(\frac{A-C+\pi+w}{2}\right)+\wedge\left(\frac{A-C+\pi-w}{2}\right),
\end{aligned}
$$


where

$$
w=\arccos \left(\frac{1+\cos B \cos B^{\prime}-\cos ^{2} A-\cos ^{2} C}{\cos B+\cos B^{\prime}+2 \cos A \cos C}\right) .
$$

Note that a different choice of $a$ induces the same formula which differs by sign. From the analyticity of the volume function of tetrahedra and from a suitable choice of dihedral angles, for example, those of a regular tetrahedron or an ideal tetrahedron, we can show that our choice is the correct one.

Example 2. The volume of $O T(A, B, C)$ can be obtained from Example 1. A tetrahedron $T(B, 2 A, B, B, 2 C, B)$ can be divided into four copies of the orthoschemes $O T(A, B, C)$. So we have the following formula, which is the same as formula (7):

$4 \operatorname{vol} O T(A, B, C)$

$$
\begin{aligned}
= & \operatorname{vol} T(B, 2 A, B, B, 2 C, B) \\
= & \wedge\left(A+\frac{w}{2}\right)-\wedge\left(A-\frac{w}{2}\right)+\wedge\left(C+\frac{w}{2}\right)-\wedge\left(C-\frac{w}{2}\right) \\
& -\wedge\left(\frac{\pi}{2}+B+\frac{w}{2}\right)+\wedge\left(\frac{\pi}{2}+B-\frac{w}{2}\right)-\wedge\left(\frac{\pi}{2}+\frac{w}{2}\right)+\wedge\left(\frac{\pi}{2}-\frac{w}{2}\right) \\
= & \wedge(A+\delta)-\wedge(A-\delta)+\wedge(C+\delta)-\wedge(C-\delta) \\
& -\wedge\left(\frac{\pi}{2}-B+\delta\right)+\wedge\left(\frac{\pi}{2}-B-\delta\right)+2 \wedge\left(\frac{\pi}{2}-\delta\right),
\end{aligned}
$$

where

$$
\begin{aligned}
\delta=\frac{w}{2} & =\frac{1}{2} \arccos \left(\frac{1+\cos 2 A \cos 2 C-2 \cos ^{2} B}{\cos 2 A+\cos 2 C+2 \cos ^{2} B}\right) \\
& =\arctan \left(\frac{\sqrt{\cos ^{2} B-\sin ^{2} A \sin ^{2} C}}{\cos A \cos C}\right) .
\end{aligned}
$$

So we can obtain formula (7) without using the Schläfli differential formula.

Remark. There is another volume formula of orthoschemes entirely different from the Lobachevsky formula (7). An orthoscheme $O T(A, B, C)$ is the same as a tetrahedron $T(\pi / 2, B, C, \pi / 2, \pi / 2, A)$. So we can deduce the following formula from the above theorem:

$$
\begin{aligned}
& \operatorname{vol} \operatorname{OT}(A, B, C) \\
& =\frac{1}{4}[\wedge(A+w)-\wedge(A-w)+\wedge(C+w)-\wedge(C-w)-2 \wedge(w) \\
& +2 \wedge\left(\frac{\pi}{4}+\frac{B+C-A-w}{2}\right)-2 \wedge\left(-\frac{\pi}{4}+\frac{B+C-A+w}{2}\right) \\
& \left.+2 \wedge\left(\frac{\pi}{4}+\frac{B+A-C-w}{2}\right)-2 \wedge\left(-\frac{\pi}{4}+\frac{B+A-C+w}{2}\right)\right]
\end{aligned}
$$

where

$$
w=\arcsin \left(\frac{\sin A \sin C}{\cos B}\right) .
$$




\section{Appendix}

As an example of the calculation of volumes obtained above, we give volumes of Coxeter tetrahedra (the dihedral angles of Coxeter tetrahedra are $\pi / n_{i}, n_{i} \in \mathbb{Z}$ ). The volumes of all Coxeter tetrahedra were calculated in [6] (see also [3] for the volumes of all Coxeter $n$-simplices) by decomposing a Coxeter tetrahedron into four orthoschemes (i.e., 28 Lobachevsky functions in general). However, formula (15) of Example 1 and formula (9) give another elegant way of calculating the volumes of all Coxeter tetrahedra.

Table 1 Volumes of noncompact tetrahedra for the corresponding Coxeter schemes, where we used formula (9).

$\mathrm{V}=\frac{1}{8} \wedge\left(\frac{\pi}{3}\right)$.
$\mathrm{V}=\frac{1}{2} \wedge\left(\frac{\pi}{3}\right)$.
$\mathrm{V}=\frac{1}{6} \wedge\left(\frac{\pi}{4}\right)$.
$\mathrm{V}=\frac{5}{16} \wedge\left(\frac{\pi}{3}\right)$.
$\mathrm{V}=\frac{3}{4} \wedge\left(\frac{\pi}{3}\right)$.
$\mathrm{V}=\frac{1}{2} \wedge\left(\frac{\pi}{4}\right)$.


Table 2 Volumes of compact tetrahedra for the corresponding Coxeter schemes, where we used formulas (7) and (15).

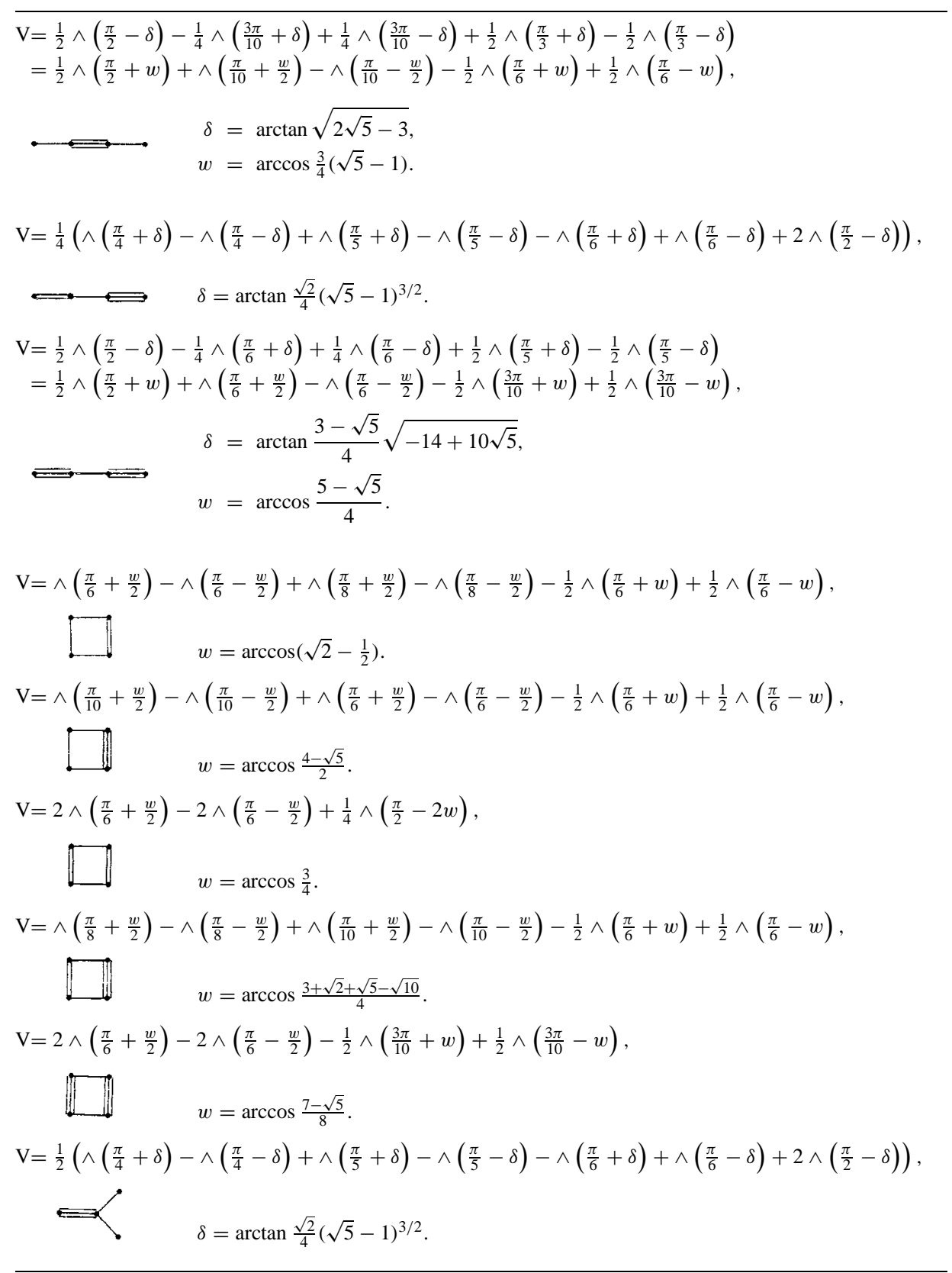




\section{References}

1. Alekseevskij, D. V., Vinberg, E. B., Solodovnikov, A. S., Geometry of space of constant curvature, in: Encyclopedia of Mathematical Science, Geometry II, Springer-Verlag, Berlin, 1993.

2. Coxeter, H. S. M., The functions of Schläfli and Lobatschefsky, Quart. J. Math., 6 (1935), 13-29.

3. Johnson, N. W., Kellerhals, R., Ratcliffe, J. G., Tschantz, S. T., The size of a hyperbolic Coxeter simplex (preprint).

4. Kellerhals, R., On the volume of hyperbolic polyhedra, Math. Ann., 285 (1989), 541-569.

5. Kellerhals, R., The dilogarithm and volumes of hyperbolic polytopes, in: Structural Properties of Polylogarithms, L. Lewin, ed., Mathematical Surveys and Monographs, vol. 37, AMS, Providence, RI, 1991, pp. 301-336.

6. Meyerhoff, R., A lower bound for the volume of hyperbolic 3-orbifolds, Duke Math. J., 57 (1988), 185-203.

7. Milnor, J., Hyperbolic geometry: the first 150 years, Bull. Amer. Math. Soc., 6 (1982), 9-24.

8. Rivin, I., Euclidean structures on simplicial surfaces and hyperbolic volume, Ann. of Math., 139 (1994), 553-580.

9. Thurston, W. P., The geometry and topology of 3-manifolds, Revised Lecture Notes, Princeton University, 1982.

10. Vinberg, E. B., The volume of Polyhedra on a sphere and in Lobachevsky space, Amer. Math. Soc. Trans. (2), 148 (1991), 15-27.

11. Vinberg, E. B., Volumes of non-Euclidean polyhedra, Russ. Math. Surveys, 48(2) (1993), 15-45.

12. Vesnin, A., On volumes of some hyperbolic 3-manifolds, Lecture Notes Series (30), Seoul National University, 1996.

Received November 6, 1997, and in revised form April 29, 1998. 